\title{
EPIDEMIOLOGIA E RISCOS ASSOCIADOS À ÚLCERAS POR PRESSÃO EM CRIANÇAS
}

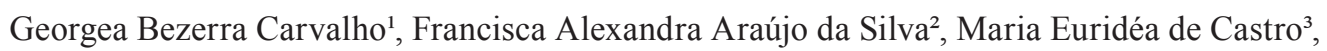
Raquel Sampaio Florêncio ${ }^{4}$

\begin{abstract}
RESUMO: Trata-se de estudo epidemiológico transversal que teve por objetivo identificar o risco e os fatores associados ao desenvolvimento de úlceras por pressão em crianças internadas em uma Unidade de Terapia Intensiva Pediátrica. Foi realizado com 40 crianças, com idades entre vinte e nove dias e oito anos; os dados foram coletados por meio de um formulário e da Escala de Braden Q. Houve homogenia em relação ao sexo, a média de idade foi de 15,3 meses. Todas as crianças foram consideradas em risco pela escala de Braden $\mathrm{Q}$, com escores altos nos itens exposição à umidade e perfusão tecidual e oxigenação. Observaram-se 26 úlceras por pressão em 17 crianças, localizadas principalmente na região occipital, e cerca de um terço foram classificadas em estágio um. Percebe-se a necessidade de mais estudos sobre a assistência às crianças criticamente doentes e que contribuam para o desenvolvimento de intervenções de prevenção desses agravos. PALAVRAS-CHAVE: Úlcera por pressão; Criança hospitalizada; Epidemiologia; Enfermagem.
\end{abstract}

\section{EPIDEMIOLOGY AND RISKS ASSOCIATED WITH PRESSURE ULCERS IN CHILDREN}

ABSTRACT: This epidemiological transversal study aimed to identify the risk of, and factors associated with, the development of pressure ulcers in children hospitalized in a Pediatric Intensive Therapy Unit. It was carried out with 40 children, aged between 29 days and eight years; the data was collected using a questionnaire and the Braden Q scale. There was homogeneity of sex and the average age was 15.3 months. All children were considered at risk on the Braden Q scale, with high scores on the following factors: exposure to moisture and tissue perfusion and oxygenation. Twentysix pressure ulcers were observed in seventeen children, localised principally in the occipital region; about a third were classified as stage 1. It is to be noted that more studies on the care of critically ill children are needed, to develop preventative interventions against these wounds.

KEYWORDS: Pressure ulcers; Hospitalized child; Epidemiology; Nursing.

\section{EPIDEMIOLOGÍA Y RIESGOS ASOCIADOS A ÚLCERAS POR PRESIÓN EN NIÑOS}

RESUMEN: Se trata de estudio epidemiológico transversal que tuvo por objetivo identificar el riesgo y los factores asociados al desarrollo de úlceras por presión en niños internados en una Unidad de Terapia Intensiva Pediátrica. Fue realizado con 40 niños, con edades entre veintinueve días y ocho años; los datos fueron recogidos por medio de un formulario y de la Escala de Braden Q. Huvo homogeneidad acerca del sexo; la media de edad fue de 15,3 meses. Todos los niños fueron considerados en riesgo por la escala de Braden Q, con scores altos en los ítems exposición a humedad y perfusión tecidual y oxigenación. Fueron observadas 26 úlceras por presión en 17 niños, ubicadas principalmente en la región occipital, y cerca de un tercio fueron clasificadas en estagio uno. Se percibe la necesidad de más estudios sobre la asistencia a los chicos criticamente enfermos que puedan contribuir para el desarrollo de intervenciones de prevención de eses agravios PALABRAS-CLAVE: Úlcera por presión; Niños hospitalizados; Epidemiología; Enfermería.

${ }^{1}$ Enfermeira. Especialista em Estomaterapia. Membro do Serviço de Estomaterapia do Hospital Infantil Albert Sabin.

${ }^{2}$ Enfermeira. Mestre em Cuidados Clínicos em Saúde. Doutoranda em Saúde Coletiva em Ampla Associação UECE/UFC/UNIFOR. Membro do Grupo de Pesquisa Epidemiologia, Cuidados em Cronicidades e Enfermagem/UECE.

${ }^{3}$ Enfermeira. Coordenadora do Curso de Especialização em Enfermagem em Estomaterapia da Universidade Estadual do Ceará - UECE. ${ }^{4}$ Enfermeira. Membro do Grupo de Pesquisa Epidemiologia, Cuidados em Cronicidades e Enfermagem - UECE. 


\section{INTRODUÇÃO}

As úlceras por pressão (UP) são consideradas importante problema de saúde pública, representando uma das principais complicações que acometem os pacientes críticos hospitalizados, prolongando a internação e dificultando a recuperação do doente ${ }^{(1)}$.

Neste contexto, os números apontam o impacto das UP nos cuidados em saúde. Em um estudo, cerca de 33\% dos doentes internados em Unidade de Terapia Intensiva (UTI) desenvolveram úlceras, associando-se a um prolongamento da internação hospitalar de até cinco vezes. Além disso, essa lesão aumenta o risco de morte para 4,5 vezes, comparando doentes com o mesmo risco prévio de mortalidade que não desenvolveram $\mathrm{UP}^{(2)}$.

Quando se trata de crianças criticamente doentes, esse risco assume proporções ainda maiores. A National Pressure Ulcer Advisory Prevention (NPUAP) ${ }^{(3)}$ estima uma taxa de prevalência de $27 \%$ em UTI pediátrica. Em pesquisa observou-se incidência de $27 \%$ de UP em crianças criticamente doentes, sendo que a maioria delas já podia ser detectada no segundo dia de internação em UTI pediátrica ${ }^{(4)}$.

Frente às evidências existentes e específicas para a prevenção desses agravos e seu tratamento nesse grupo, repleto de peculiaridades e, considerando-se que a maioria dos produtos e equipamentos são desenvolvidos para os adultos, consideramos relevante este tema para estudo.

A European Pressure Ulcer Advisory Panel (EPUAP) ${ }^{(5)}$ ressalta a falta de informação sobre o tema e considera que as diferenças existem em todos os sentidos e se referem ao estado de maturação da pele (dependendo da idade da criança), às regiões onde as UP são frequentemente identificadas, ao tamanho do corpo em relação ao equipamento médico, movimentos, expressões e manifestações da dor, entre outros. Assim, entende-se ser essencial que as diferenças entre adultos e crianças sejam consideradas, tendo uma política de prevenção específica para essa população.

A identificação e o tratamento precoces permitem uma redução significativa dos custos, previnem a progressão e aceleram a regeneração da UP. Estima-se que a adoção de medidas preventivas reduz o risco entre 25 a $50 \%{ }^{(2)}$.

Diante da importância da prevenção desses agravos, destaca-se a necessidade de avaliação do risco para o desenvolvimento de UP, o qual deve ser feito sistematicamente, não só na admissão do doente, como em todas as fases do seu tratamento.

Com base no que foi exposto, o estudo consistiu em conhecer a epidemiologia e identificar o risco e os fatores associados à ocorrência de UP em crianças internadas em uma UTI pediátrica.

\section{METODOLOGIA}

Trata-se de uma pesquisa epidemiológica transversal, realizada em um hospital público de referência em pediatria, localizado em Fortaleza-Ceará, Brasil. A unidade do estudo foi a UTI pediátrica, que recebe em sua maioria crianças com problemas clínicos e, em menor proporção, cirúrgicos.

A população do estudo foi composta por crianças internadas na referida unidades; o critério de inclusão foi a admissão da criança na UTI há pelo menos 24 horas; o critério de exclusão foi a detecção de UP préexistente na admissão. A amostra compreendeu 40 crianças com idade entre 2 meses e 8 anos.

Os dados foram coletados nos meses de maio, junho e julho de 2007. Inicialmente, foi aplicado um formulário, especificamente elaborado para o estudo e previamente testado, composto de itens referentes aos dados demográficos e clínicos, tendo como fonte de informação os prontuários e a observação clínica. Posteriormente, utilizou-se a Escala de Braden Q para classificar o risco para UP, modificada para uso em pediatria, seguido do exame clínico. Neste estudo, a escala foi aplicada na primeira avaliação e, semanalmente, até a alta da criança. A diferenciação das lesões em estágios foi realizada conforme a classificação proposta pela NPUAP ${ }^{(6)}$. O exame da pele foi realizado três vezes por semana nas primeiras duas semanas, e semanalmente até a alta da UTI.

A escala em questão ainda não havia sido validada em nosso país por ocasião deste estudo, portanto, para ser utilizada, foi solicitada autorização das autoras para que essa fosse traduzida por um tradutor credenciado, e então aplicada neste estudo.

Os dados obtidos foram organizados e analisados estatisticamente, sendo utilizado o programa Predctive Analitics Software for Windows (PASW) versão 17.0. Utilizou-se o cálculo de frequência simples e relativa para as variáveis nominais e a média e desvio padrão para variáveis contínuas. A relação entre tipo de medicamento e presença de UP, e entre presença de UP e risco para UP, foi verificada pelo teste qui-quadrado de Pearson, tendo sido considerado o valor de $\mathrm{p}<0,05$ para a significância estatística.

O projeto foi enviado para apreciação do Comitê de Ética em Pesquisa do hospital do estudo, o qual emitiu parecer autorizando sua realização sob protocolo n. 86/06. Foram respeitados os princípios éticos para pesquisas envolvendo seres humanos, de acordo com as normas estabelecidas pela resolução n. 196/96 do Conselho Nacional de Saúde ${ }^{(7)}$. 


\section{RESULTADOS}

Inicialmente, foram descritas, conforme a tabela 1 , as características demográficas das crianças do estudo.

Com relação ao sexo, observou-se homogeneidade na amostra; com relação à coloração da pele, a maioria dos sujeitos são de cor branca e desses, $11(40 \%)$ apresentaram UP, enquanto que entre os não brancos 4 (31\%) apresentaram a lesão. A média de idade foi de, aproximadamente, 15,3 meses $( \pm 7,1)$, com idade mínima de 2 meses e máxima de 84 meses; a maioria dos pacientes era procedente da capital.

Tabela 1- Distribuição da amostra de crianças internadas em UTI conforme dados demográficos. Fortaleza, 2007

\begin{tabular}{|c|c|c|}
\hline Variável & $f$ & $\%$ \\
\hline \multicolumn{3}{|l|}{$\overline{\text { Sexo }}$} \\
\hline Masculino & 19 & 47,5 \\
\hline Feminino & 21 & 52,5 \\
\hline \multicolumn{3}{|l|}{ Idade } \\
\hline 2 meses a 1 ano & 32 & 80 \\
\hline 2 a 5 anos & 5 & 12,5 \\
\hline Acima de 5 anos & 3 & 7,5 \\
\hline \multicolumn{3}{|l|}{ Cor } \\
\hline Branco & 27 & 67,5 \\
\hline Não branco & 13 & 32,5 \\
\hline \multicolumn{3}{|c|}{ Presença de acompanhante } \\
\hline Sim & 26 & 65 \\
\hline Não & 14 & 35 \\
\hline \multicolumn{3}{|l|}{ Procedência } \\
\hline Fortaleza & 19 & 47,5 \\
\hline Outras cidades & 21 & 52,5 \\
\hline Total & 40 & 100 \\
\hline
\end{tabular}

Observa-se que $32(80 \%)$ crianças tinham idade menor que 1 ano, fato que pode ter sido influenciado pelo tamanho do berço da UTI, que mede $1 \mathrm{~m}$ e $40 \mathrm{~cm}$. Dessa forma, quando uma criança maior necessita de leito de UTI ela é enviada para a UTI pós-operatória, no mesmo hospital, a qual tem 8 leitos, sendo 6 deles de camas.

Além das características demográficas, os dados clínicos também foram avaliados. Em relação ao peso, obteve-se uma média de 7,4 kg $( \pm 1,4)$, com peso mínimo de 2,7 kg e máximo de $20 \mathrm{~kg}$; além disso, 32 (80\%) crianças tinham peso inferior a $12 \mathrm{~kg}$. O tempo médio de internação, entre as que apresentaram UP, foi de 21,4 dias, variando entre 5 e 45 dias, sendo que 7 (53\%) estavam internadas há mais de 15 dias. Entre as que não apresentaram UP, o tempo médio de internação foi de 7,7 dias.

Foi investigada também a presença, ou não, de acompanhantes; 26 (65\%) tinham acompanhantes na maior parte de tempo. Entre as 17 crianças que apresentaram UP, apenas cinco não tinham acompanhante.

Com relação à presença de dispositivos médicos, cinco $(19,2 \%)$ lesões foram detectadas em asa de nariz, como consequência da utilização de sondas, tanto nasogástrica como nasoenteral.

Na avaliação da Pressão Arterial Média (PAM), foi encontrado que, dos 7 pacientes considerados hipotensos, três apresentaram UP. De acordo com a escala utilizada, o paciente foi considerado hipotenso quando a PAM esteve menor que $50 \mathrm{mmHg}$.

$\mathrm{Na}$ avaliação dos exames laboratoriais destacou-se que $7(17,5 \%)$ crianças apresentaram albumina inferior a $3 \mathrm{mg} / \mathrm{dl}$, e entre estas, três apresentaram UP. Em 16 (40\%) dos casos não foi encontrada essa informação.

De acordo com a tabela 2, de modo geral, a amostra da pesquisa apresentou escores altos apenas nos itens "exposição à umidade" e "perfusão tecidual e oxigenação", obtidos após aplicação da Escala de Braden Q.

Tabela 2- Escores obtidos nos itens da escala de Braden Q em crianças internadas em UTI.. Fortaleza, 2007

\begin{tabular}{|c|c|c|c|c|c|c|c|c|c|}
\hline \multirow{3}{*}{ Escala de Braden Q (itens) } & \multicolumn{8}{|c|}{ Escores } & \multirow{3}{*}{ Total } \\
\hline & \multicolumn{2}{|c|}{1} & \multicolumn{2}{|c|}{2} & \multicolumn{2}{|c|}{3} & \multicolumn{2}{|c|}{4} & \\
\hline & $f$ & $\%$ & $f$ & $\%$ & $f$ & $\%$ & $f$ & $\%$ & \\
\hline Mobilidade & 26 & 15,38 & 43 & 22,99 & 18 & 13,53 & 0 & 0 & 87 \\
\hline Atividade física & 85 & 50,30 & 2 & 1,07 & 0 & 0 & 0 & 0 & 87 \\
\hline Percepção sensorial & 15 & 8,88 & 54 & 28,88 & 18 & 13,53 & 0 & 0 & 87 \\
\hline Exposição a umidade & 0 & 0 & 0 & 0 & 1 & 0,75 & 86 & 71,67 & 87 \\
\hline Fricção e cisalhamento & 0 & 0 & 35 & 18,72 & 52 & 39,1 & 0 & 0 & 87 \\
\hline Estado nutricional & 38 & 22,49 & 34 & 18,18 & 15 & 11,28 & 0 & 0 & 87 \\
\hline Perfusão tecidual e oxigenação & 5 & 2,96 & 19 & 10,16 & 29 & 21,8 & 34 & 28,33 & 87 \\
\hline Total & 169 & 100 & 187 & 100 & 133 & 100 & 120 & 100 & 609 \\
\hline
\end{tabular}

Cogitare Enferm. 2011 Out/Dez; 16(4):640-6 
Na subescala "mobilidade" 38,4\% das avaliações também indicaram escores baixos, entre um e dois. Na subescala "atividade física", em 97,7\% das avaliações os pacientes foram classificados como "acamados", pois apresentavam comprometimento clínico que os impedia até mesmo de estar nos braços do cuidador. Com relação ao item "percepção sensorial", em 37,8\% das avaliações os escores foram entre um e dois, fato que pode ser explicado pelo grande número de pacientes em uso de sedativo contínuo.

Com relação à subescala "exposição à umidade", apenas uma criança, em uma avaliação, foi considerada como "ocasionalmente úmido", em todas as outras avaliações os pacientes foram classificados como "raramente úmido". Apesar das crianças, de uma forma geral, serem consideradas incontinentes, as fraldas são trocadas em horários de rotina e durante as avaliações a pele estava quase sempre enxuta.

Na subescala "fricção e cisalhamento", também não foram observadas diferenças importantes nas avaliações. Nenhuma criança foi classificada como "sem problema aparente". Além disso, como 95\% da amostra recebia sedativos em período contínuo, as crianças apresentavam restrição dos movimentos, diminuindo também as forças de fricção.

Com relação à subescala "estado nutricional", observou-se que, na primeira avaliação, no ato da admissão, 33 (82,5\%) das crianças foram incluídos no item "péssimo". Isso é explicado pelo fato de que, ao entrar na UTI, o paciente quase sempre está instável clinicamente e fica sob observação rigorosa, em dieta zero.

Por outro lado, o item "excelente" não foi encontrado nenhuma vez, pois em se tratando de pacientes que necessitam de UTI, mesmo que ocorra melhora clínica, na maioria das vezes, encontra-se com algum comprometimento do estado geral que pode restringir de alguma forma a alimentação.

Os achados do estudo informaram que 17 crianças $(42,5 \%)$ desenvolveram 26 úlceras por pressão, conforme a tabela 3 .

A principal localização das UP foi a região occipital. Das 26 úlceras encontradas, 25 foram detectadas na cabeça e, dessas, 14 lesões estavam na região occipital.

Com relação ao estágio das UP, foi possível observar que $10(38,5 \%)$ desenvolveram UP em estágio I, $7(27,0 \%)$ foram classificadas como estágio II e $10(38,5 \%)$ apresentaram tecido necrótico ou escara (estágio III).

Neste estudo, encontrou-se que $12(70,6 \%)$ das lesões foram detectadas na primeira semana de internamento, entretanto, nenhuma delas foi detectada na primeira avaliação da pele. Além disso, observou-se que a precocidade com a qual a lesão aparecia era proporcional à piora clínica do doente.

Em relação aos medicamentos utilizados, sabe-se que alguns podem contribuir para o desenvolvimento de UP, sobretudo, se forem de uso contínuo. Apenas três crianças não faziam uso de sedativo contínuo; com relação aos medicamentos vasoativos, 17 fizeram uso de drogas dessa natureza, e dessas 10 (33,3\%) apresentaram UP. No entanto, não foi encontrada associação estatística significativa entre as variáveis "medicamento usado" e "presença de UP" nos pacientes pesquisados $(\mathrm{p}=0,298)$.

De acordo com a Escala de Braden Q, todos os pacientes foram considerados como em risco para desenvolver UP; 30 crianças da amostra (75\%) foram consideradas como de alto risco. A relação entre "risco de UP" e "presença de UP" foi verificada, no entanto, não foi encontrada associação estatística significativa entre tais variáveis $(\mathrm{p}=0,196)$.

Tabela 3- Região corporal com úlcera por pressão e estágio da lesão em crianças internadas em UTI. Fortaleza, 2007

\begin{tabular}{|c|c|c|c|c|c|c|c|}
\hline \multirow[t]{3}{*}{ Região } & \multicolumn{6}{|c|}{ Classificação da UP } & \multirow[t]{3}{*}{ Total } \\
\hline & \multicolumn{2}{|c|}{ Estágio I } & \multicolumn{2}{|c|}{ Estágio II } & \multicolumn{2}{|c|}{$\begin{array}{l}\text { Suspeita lesão tissular } \\
\text { profunda }\end{array}$} & \\
\hline & $f$ & $\%$ & $f$ & $\%$ & $f$ & $\%$ & \\
\hline Região occipital & 2 & 20,0 & 2 & 33,33 & 10 & 100 & 14 \\
\hline Pavilhão auricular & 4 & 40,0 & 2 & 33,33 & 0 & 0 & 6 \\
\hline Asa de nariz & 3 & 30,0 & 2 & 33,33 & 0 & 0 & 5 \\
\hline Maléolo & 1 & 10,0 & 0 & 0 & 0 & 0 & 1 \\
\hline Total & 10 & 100 & 6 & 100 & $\mathbf{1 0}$ & 100 & 26 \\
\hline
\end{tabular}




\section{DISCUSSÃO}

Existem algumas evidências sugerindo que a coloração da pele está associada com a incidência das UP, porém não está claro de que forma essa interação acontece ${ }^{(8)}$. Na literatura há consenso quanto à resistência da pele negra à agressão externa, causada pela umidade e fricção. No entanto, vale ressaltar que existe certa dificuldade em identificar as UP em estágio I nos indivíduos de cor negra, fato que pode contribuir para subestimar o número de UP nessa população(1).

Outro fator importante a ser destacado é o tempo de internação. Em pesquisa realizada comparando crianças gravemente doentes, com e sem UP, um dos fatores de risco para o desenvolvimento dessas lesões foi o tempo de internação superior a 96 horas $^{(9)}$.

A literatura pesquisada destaca a importância do cuidador; crianças que não têm acompanhante para mobilizá-las ou reposicioná-las, ou crianças que não têm cuidadores educados quanto à importância do reposicionamento, são colocadas em alto risco quando comparadas a crianças que têm um cuidador treinado ${ }^{(10)}$.

Em estudo realizado ${ }^{(11)}$, no qual foi avaliado a localização, incidência e fatores associados a UP em unidades de cuidado intensivo pediátrico, 27 lesões foram detectadas como causadas por dispositivos médicos, entre os quais sensores para determinar a $\mathrm{SaO}_{2}$, máscaras de Bipap, tubo endotraqueal, traqueostomia, cateteres e talas.

Outra pesquisa verificou que, em cerca de $50 \%$ $(\mathrm{n}=27)$ das crianças estudadas, as UP puderam ser relacionadas com o uso de equipamentos sobre a pele causando pressão ou fricção e cisalhamento ${ }^{(12)}$.

Níveis pressóricos baixos colaboram para que haja o fechamento dos capilares e estão associados à ocorrência de UP. A hipotensão pode diminuir o fluxo sanguíneo para os órgãos vitais e reduzir a tolerância da pele a lesões ${ }^{(13)}$.

Os sedativos e analgésicos, ao reduzir a sensação de dor, acabam por reduzir a mobilidade; os agentes hipotensores podem afetar o fluxo sanguíneo reduzindo a perfusão dos tecidos, o que torna mais suscetíveis à pressão(1).

A pouca literatura sobre o tema informa uma taxa de incidência entre 17 e $25 \%$ na população pediátrica criticamente doente ${ }^{(8)}$. Em outro estudo, verificou-se uma taxa de incidência de úlceras por pressão de $27 \%$ em um grupo de pacientes pediátricos criticamente doentes ${ }^{(11)}$.
Nossos dados corroboram com estudos nos quais cerca de 30\% das UP foram detectadas na cabeça, incluindo a região occipital e outras regiões como orelha e nariz ${ }^{(11,14)}$. Uma preocupação concernente às UP na região occipital se deve ao fato de sua ocorrência estar ligada ao desenvolvimento de cicatriz e alopecia em crianças com comprometimento da oxigenação e perfusão, ou naquelas com complicação do uso da oxigenação de membrana extracorpórea ${ }^{(10)}$.

Em estudo multissistêmico ${ }^{(14)}$, com 1064 crianças hospitalizadas nos EUA, informou-se que $92 \%$ das UP eram de estágio I. Em estudo semelhante ${ }^{(11)} 70 \%$ das UP eram estágio I, $27 \%$ de estágio II e apenas $3 \%$ de estágio III.

No que diz respeito ao período em que a úlcera foi detectada, alguns estudos ressaltam um tempo mínimo para o desenvolvimento das lesões. Estudo realizado em UTI pediátrica revelou que $57 \%$ de todas as úlceras foram detectadas durante a primeira avaliação da pele, logo no segundo dia de internamento na unidade ${ }^{(8)}$.

No que diz respeito à ocorrência do risco, ou não, a literatura mostra que crianças que tenham o total de escores da Braden Q em torno 25 são consideradas como em baixo risco para desenvolver UP; escores em torno de 21 indicam risco moderado e escores em torno de 16 apontam alto risco ${ }^{(15)}$. A utilização de escalas tem como objetivo a intervenção na prevenção de UP. Existem algumas evidências de que programas de prevenção, guiados por avaliação de risco, podem simultaneamente reduzir a incidência institucionalizada das UP em até $60 \%$ e derrubar os custos em prevenção ${ }^{(16)}$.

Apesar da importância dada à utilização de escalas para avaliar o risco de UP, neste estudo a maioria das crianças que estava em alto risco não apresentaram úlcera. Com base nisso, entende-se que a simples aplicação da escala pode fornecer resultados contraditórios, portanto, sua utilização deve estar associada ao julgamento clínico do enfermeiro, o qual deve considerar a condição do paciente, o contexto de sua doença e a influência desses fatores na ocorrência, ou não, da UP.

De fato, existem críticas quanto a algumas escalas de risco. Pacientes que foram avaliados como sendo de risco não desenvolveram UP, enquanto outros, avaliados como não sendo de risco, a desenvolveram ${ }^{(17)}$.

Em estudo mostrou o quão é evidente a necessidade da realização de educação continuada com toda a equipe de enfermagem, tendo como estratégia incorporar novas tecnologias à práticas do cuidado frente ao cuidado de feridas ${ }^{(18)}$. 
Nesse caso, destacamos a escassez de estudos envolvendo a população pediátrica e a utilização de escalas de risco para UP. Portanto, mais investigações devem ser realizadas com o intuito de identificar a relação entre esses temas. Ressalta-se que a Escala de Braden Q foi traduzida para lingua portuguesa com adaptação cultural e validação, e cujos resultados foram publicados recentemente ${ }^{(19)}$.

\section{CONCLUSÃO}

As crianças internadas por longos períodos na UTIP têm maior chance de desenvolver UP, podendo o período ser considerado como fator de risco. Quanto à Escala de Braden Q, de modo geral, a amostra desta pesquisa apresentou escores altos apenas nos itens "exposição à umidade" e "perfusão tecidual e oxigenação".

Houve ocorrência importante de UP nas crianças pesquisadas, fato que vai contra pressupostos que afirmam relativa raridade desse agravo nessa população. Contudo, o presente estudo se soma às tentativas de contribuir para um maior conhecimento acerca da ocorrência de UP em crianças criticamente doentes, dada a importância desse tema e a escassez de pesquisas na área.

Desta forma, destaca-se a necessidade da realização de outras pesquisas envolvendo essa temática no nosso país, para que se possa confirmar, ou não, os achados desta pesquisa.

\section{REFERÊNCIAS}

1. Blanes L, Duarte IS, Calil JÁ, Ferreira LM. Avaliação clínica e epidemiológica das úlceras de pressão em pacientes internados no Hospital São Paulo. Rev. Assoc. Med. Bras. São Paulo. 2004; 50(2):182-7

2. Rocha JA, Miranda MJ, Andrade MJ. Abordagem terapêutica das úlceras de pressão - intervenções baseadas na evidência. Acta Medica Portuguesa, Porto. 2006;19:19-29.

3. National Pressure Ulcer Advisory Prevention (NPUAP). Statement on pressure ulcer prevention. 1992. [acesso em 22 jul. 2006]. Disponível em: http://www.npuap. org/position1.htm.

4. Curley MA, Razmus IS, Roberts KE, Wypit D. Predicting pressure ulcer risk in pediatric patients: The Braden Q Scale. Nurs Res. 2003;52(1):22-33.
5. European Pressure Ulcer Advisory Panel (EPUAP). Abstracts from the Sixth EPUAP Open Meeting. pressure ulcers in children. Budapest. 2002. [acesso em 22 jul 2006]. Disponível em: http://www.epuep.org/ review5_1/page $5 \mathrm{~h} . \mathrm{html}$.

6. National Pressure Ulcer Advisory Panel (NPUAP). Updated staging system. Pressure ulcer stages revised by NPUAP. 1989. [acesso em 30 jun 2007]. Disponível: http://www.npuap.org/pr2.htm.

7. Ministério da Saúde (BR). Conselho Nacional de Saúde. Diretrizes e normas regulamentadoras de pesquisa envolvendo seres humanos. Resolução n. 196, de 10 de outubro de 1996. Brasília; 1996.

8. Carnevale FA. Pressure ulcers in pediatric critical care: Examining the evidence. Pediatric Critical Care Medicine. 2003;4(3):383-4.

9. Cord S, Elvain V, Sachdeva R, Schwartz P, Jefferson LS. Risk factors associated with pressure ulcers in the pediatric intensive care unit. J Wound Ostomy Continence Nurs. 2004;31(4):173-83.

10. Dixon M, RatliffC. Pediatric pressure ulcer prevalence: one hospital's experience. Ostomy Wound Management. 2005;51(6):44-55.

11. Curley MA, Quigley SA, Lin M. Pressure Ulcers in pediatric intensive care: incidence and associated factors. Pediatric Clinical Care Medical. 2003;4(3):28490.

12. Willock J, Harris C, Harrison J, Poople C. Identifying the characteristics of children with pressure ulcers. Nurs Times. 2005;101(11):40-3.

13. Fernandes LM, Úlcera de pressão em pacientes críticos hospitalizados: Uma revisão integrativa da literatura [dissertação]. Ribeirão Preto (SP): Escola de Enfermagem de Ribeirão Preto, Universidade de São Paulo; 2000.

14. Mc Lane KM, Bookout K, Mc Cords S, Mc Cain J, Jefferson LS. The 2003 National pediatric pressure ulcer and skin breakdown prevalence survey: a multisite study. J Wound Ostomy Continence Nurs. 2004;31(4):168-78.

15. Gray M. Which pressure plcer risk scales are valid and reliable in a pediatric population? J Wound Ostomy Continence Nurs. 2004;31(4):157-60.

16. Braden, BJ. Risk assessment in pressure ulcer 
prevention. In: Krasner D, Rodeheaver GR, Sibbald RG, editors. Chronic wound care: A clinical source book for healthcare professionals. 3th. Wayne: HMP Communications; 2001. p.641-51.

17. Dealy C. O tratamento de pacientes com feridas crônicas. In: Dealy C. Cuidando de feridas: um guia para as enfermeiras. São Paulo: Atheneu; 2001. p. 287-98.

18. Martins D, Soares F. Conhecimento sobre prevenção e tratamento de úlceras de pressão entre trabalhadores de enfermagem em um hospital de Minas Gerais. Cogitare Enferm. 2008;13(1):83-7.

19. Maia ACAR, Pellegrino DMS, Blanes L, Dini GM, Ferreira LM. Tradução para a língua portuguesa e validação da escala de Braden Q para avaliar o risco de úlcera por pressão em crianças. Rev Paul Pediatr.[Internet]. 2011;29(3) [acesso em $01 \mathrm{dez} 2011$ ]. Disponível: http://www.scielo.br/scielo.php?script=sci arttext\&pid=S0103-05822011000300016\&lng=en. http://dx.doi.org/10.1590/S0103-05822011000300016. 